\title{
Heparin and commercial bothropic antivenom against the paralyzing effect of Bothrops jararacussu snake venom
}

\section{Rostelato-Ferreira S (1), Rodrigues-Simioni L (1), Oshima-Franco Y (1, 2)}

(1) Department of Pharmacology, School of Medical Sciences, State University of Campinas, Campinas, São Paulo State, Brazil; (2) Pharmacy Course, University of Sorocaba, Sorocaba, São Paulo State, Brazil.

\begin{abstract}
The crude venom of Bothrops jararacussu (Bjssu) is known to induce muscular paralysis in vitro. Many studies have shown that various substances, including heparin, neutralize the damage caused by snake venom. In the present study, the ability of heparin (Hep) and commercial bothropic antivenom (CBA) to neutralize neuromuscular effects of Bjssu venom, at different time-points, was analyzed. Mouse phrenic nerve-diaphragm preparation was used through a conventional myographic technique, following five different protocols: Group 1 was incubated with Bjssu $(40 \mu \mathrm{g} / \mathrm{mL})$ without any other treatment; Groups 2 and 3 were pretreated with heparin $(1 \mu \mathrm{L} / \mathrm{mL})$ and CBA $(120 \mu \mathrm{L} / \mathrm{mL})$, respectively, for 15 minutes before venom addition; Group 4 after $50 \%$ neuromuscular blockade induced by Bjssu crude venom received $1 \mu \mathrm{L} / \mathrm{mL}$ of heparin while Group 5 received a mixture of Hep:CBA:Bjssu. Control preparations (Tyrode) were treated with Hep and CBA (mean \pm SEM; $n=3-6$ ). After 120 minutes of venom incubation, Group 1 preparations presented twitch-tension of $12 \pm 2 \%$. However, in Groups 2 and 3, the neutralizations were $92 \pm 1.9 \%$ and $81 \pm 6 \%$, respectively. The heparin addition, after $50 \%$ neuromuscular blockade by Bjssu, produced $40 \pm 6 \%$ muscular response after 120 minutes of incubation. Hep:CBA:Bjssu mixture displayed a protective effect of $84 \pm$ $10 \%$ against venom action. In conclusion, heparin and commercial bothropic antivenom efficiently neutralized the neurotoxic effects caused by $B$. jararacussu crude venom, even at different incubation time-points.
\end{abstract}

KEY WORDS: Bothrops jararacussu, heparin, neutralization, nerve-muscle preparation.

CONFLICTS OF INTEREST: There is no conflict.

FINANCIAL SOURCE: CNPq.

\section{CORRESPONDENCE TO:}

SANDRO ROSTELATO-FERREIRA, Laboratório de Farmacologia da Junção Neuromuscular, Departamento de Farmacologia, FCM, UNICAMP, Caixa Postal 6111, Campinas, SP, 13.083-970, Brasil. Phone +55 193521 9533. Email: srostelato@gmail.com. 


\section{INTRODUCTION}

In Brazil venomous snakebite accidents represent an important public health problem. About $90 \%$ of them are caused by Bothrops snakes and these cases are distributed among different areas of the country $(1,2)$.

Bothrops jararacussu is one of the largest species, in relation to size, of crotalid snakes found in southern Brazil, northern Argentina and eastern Paraguay. Its venom has been studied since the early 1900s (3), and produces envenomation signs and symptoms similar to other Bothrops species (4).

Although $B$. jararacussu venom provokes no pronounced neurotoxic effects in vivo, it abolishes, both directly and indirectly, evoked muscle contractions in mouse, chick and frog neuromuscular preparations $(5,6)$. This activity has been attributed to the presence of phospholipases and myotoxins (6-8). Bothropstoxin-I (BthTX-I), the major myotoxin in $B$. jararacussu venom, is a $13.7 \mathrm{kDa}$ single chain polypeptide capable of forming dimers (6-9).

Serum therapy has been used worldwide for decades to treat patients after snakebites (10). However, in the last few years, many other strategies have been used to minimize the damages caused by these toxins (11-13). For example heparin, a natural anticoagulant molecule that, due to its acidity, can neutralize basic phospholipases $A_{2}$, is under intense investigation as an alternative treatment (14).

In the present study, different types of heparin treatment were examined to prevent the neurotoxic activity of $B$. jararacussu crude venom. Commercial bothropic antivenom was also used on mouse neuromuscular preparation to enable further comparative analysis.

\section{MATERIALS AND METHODS}

\section{Materials}

B. jararacussu venom (Bjssu) was provided by the Butantan Institute (São Paulo, São Paulo state, Brazil); heparin (Liquemine ${ }^{\circledR}, 25,000 \mathrm{IU} / \mathrm{mL}$ - B 1019) was purchased from Roche (Brazil); commercial bothropic antivenom (CBA) (FUNED, batch 030611-12) was kindly donated by the Regional Office of Health (ERSA) at Piracicaba (São Paulo state, Brazil). 


\section{Animals}

Adult male Swiss white mice (25-30 g) were supplied by the Animal Services Unit of the State University of Campinas (UNICAMP) and maintained in a temperaturecontrolled room at $25 \pm 3^{\circ} \mathrm{C}$ on a 12 hour light/dark cycle, with free access to food and water ad libitum. This experimental protocol was approved by the University Committee for Ethics in Animal Experimentation (CEEA - Institute of Biology, UNICAMP, protocol 792-1) and the experiments were done in accordance with the guidelines established by the Brazilian College for Animal Experimentation (COBEA).

\section{Mouse Phrenic Nerve-Diaphragm Preparation}

Mice were deeply anesthetized with halothane (Cristália, Brazil) and killed by exsanguination to enable obtainment of phrenic nerve-diaphragm preparations (PND) (15). The diaphragm was removed, mounted under a tension of $5 \mathrm{~g}$ in a $5 \mathrm{~mL}$ organ bath containing Tyrode solution $\left(\mathrm{NaCl} 137 \mathrm{mM}, \mathrm{KCl} 2.7 \mathrm{mM} ; \mathrm{CaCl}_{2} 1.8 \mathrm{mM}\right.$; $\mathrm{MgCl}_{2}$ $0.49 \mathrm{mM} ; \mathrm{NaH}_{2} \mathrm{PO}_{4} 0.42 \mathrm{mM}$; $\mathrm{NaHCO}_{3} 11.9 \mathrm{mM}$ and glucose $11.1 \mathrm{mM}$ ) (pH 7.4, $37 \mathrm{C}$ ) and aerated with $95 \% \mathrm{O}_{2}$ and $5 \% \mathrm{CO}_{2}$. Supramaximal stimuli $(4 \mathrm{x}$ thresholds, $0.1 \mathrm{~Hz}, 0.2 \mathrm{~ms}$ ) delivered from a Grass S48® stimulator (Astro-Med Inc., USA) were applied to the nerve through bipolar electrodes. Isometric muscle tension was recorded by a force displacement transducer (Load Cell BG-10 GM®, Kulite Semiconductor Products Inc., USA) coupled to a physiograph (Gould, model RS 3400) via a Gould universal amplifier (both from Gould Inc., Recording Systems Division, USA). The preparations were allowed to stabilize for at least 20 minutes before addition of either treatment.

Protocols carried out: Bjssu crude venom (40 $\mu \mathrm{g} / \mathrm{mL})$; heparin $(1 \mu \mathrm{L} / \mathrm{mL})$ or antivenom $(120 \mu \mathrm{L} / \mathrm{mL})$ was pretreated for 15 minutes before the venom addition; heparin addition after $50 \%$ twitch-tension blockade was induced by Bjssu crude venom; mixture (heparin:antivenom - 1:120 $\mathrm{LL} / \mathrm{mL}$ ) and venom $(40 \mu \mathrm{g} / \mathrm{mL}$ ) were added. All experiments were compared to the three controls (Tyrode solution, heparin and antivenom alone).

\section{Statistical analysis}

All data were presented as mean \pm SEM of the number of experiments done (three to eight repetitions each protocol). Statistical comparisons were accomplished using ANOVA (repeated measures) with $p<0.05$ indicating significance. 


\section{RESULTS}

\section{Neuromuscular Activity of Mouse Phrenic Nerve-Diaphragm Preparation (PND) Submitted to Different Treatments}

Figure 1 shows the results after submitting the nerve-diaphragm preparations to Tyrode solution, heparin $(1 \mu \mathrm{L} / \mathrm{mL})$, antivenom $(120 \mu \mathrm{L} / \mathrm{mL})$ and $B$. jaracussu crude venom $(40 \mu \mathrm{g} / \mathrm{mL})$. Note that the preparation treated with commercial antivenom displayed facilitative effect (increase of twitch-tension amplitude). Significant differences between treated and control results were observed $(p<0.05)$.

Bjssu venom produced 50\% neuromuscular blockade in $41.5 \pm 2.6$ minutes $(n=6)$, which is ideal for studying the neutralizing capacity of heparin and antivenom. Indeed, after 120 minutes of incubation, the venom showed $12 \pm 2 \%(n=6)$ contractile response.

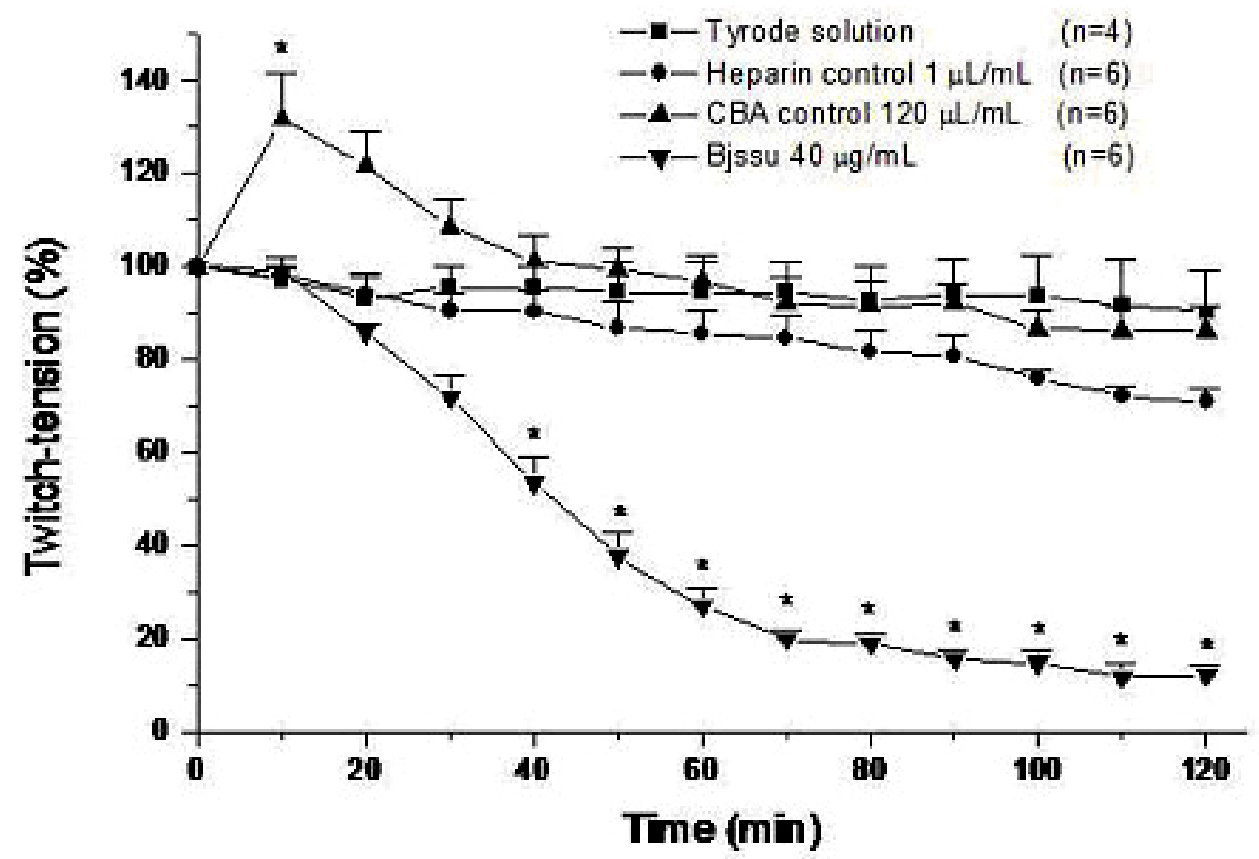

Figure 1. Neuromuscular effects of Tyrode solution, heparin, antivenom and Bjssu crude venom on the indirectly stimulated mouse phrenic nerve-diaphragm preparations. The points represent the mean \pm SEM of experiments indicated in parentheses. ${ }^{*} p<0.05$ 
Contractile Response of Pretreated PND after Bjssu Venom Addition at Different Time-Points

At the end of 120 minutes of observation, pretreated preparations (heparin or antivenom for 15 minutes), before venom addition (Figure $2-A$ and $B$ ), displayed respective twitch-tensions of $92 \pm 1.9 \%(n=3)$ and $81 \pm 6 \%(n=3)$. In the second protocol the heparin addition after the venom-induced $50 \%$ blockade (Figure $2-\mathrm{C}$ ) maintained the neuromuscular response in $40 \pm 6 \%(n=4)$. The Bjssu:Hep:CBA mixtures produced (Figure $2-$ D) a twitch-tension of $84 \pm 10 \%(n=3)$ against the venom action.

In all protocols the protective effect of heparin against the toxic action of $B$. jararacussu venom was observed. 

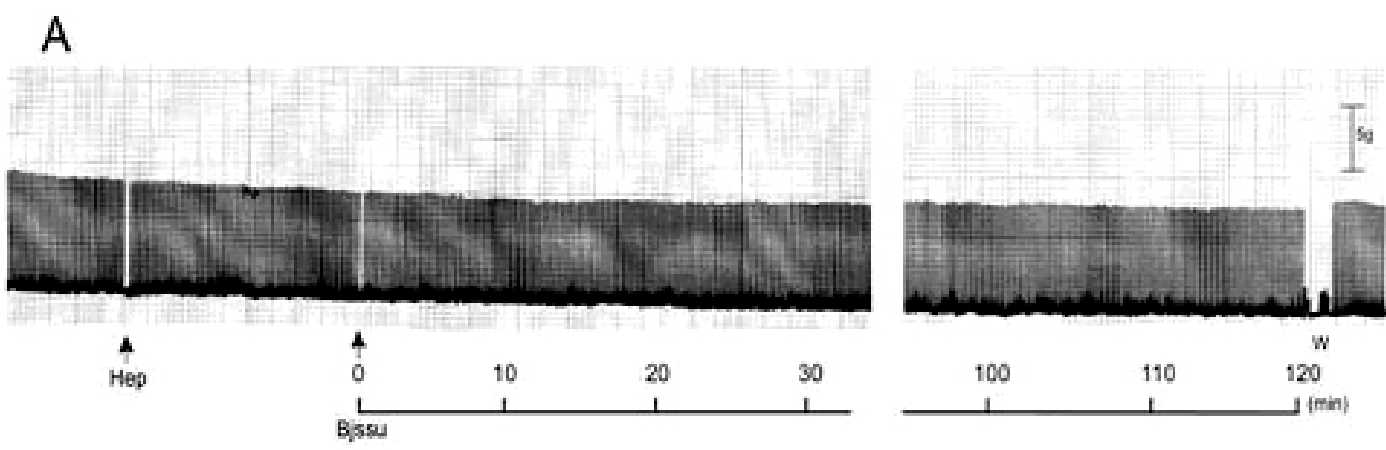

B
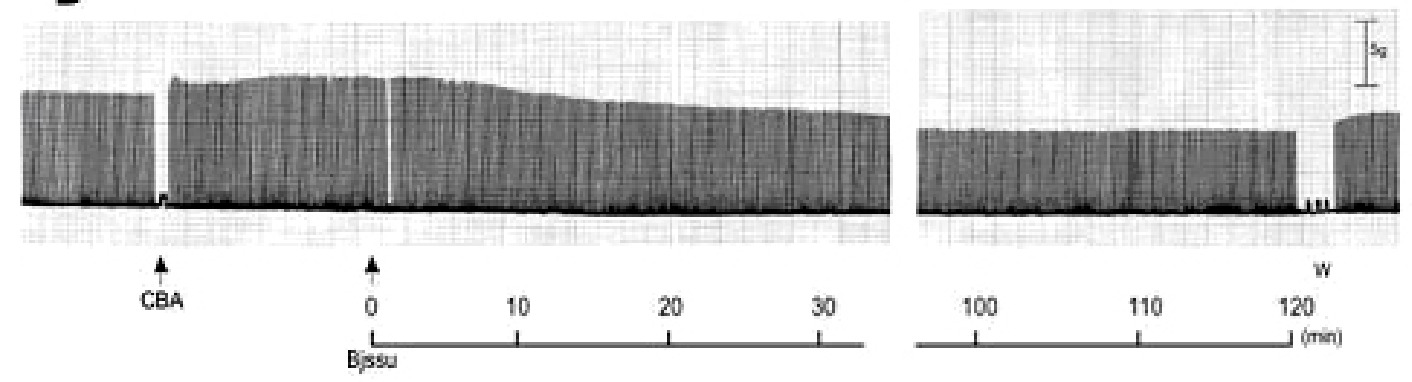

C
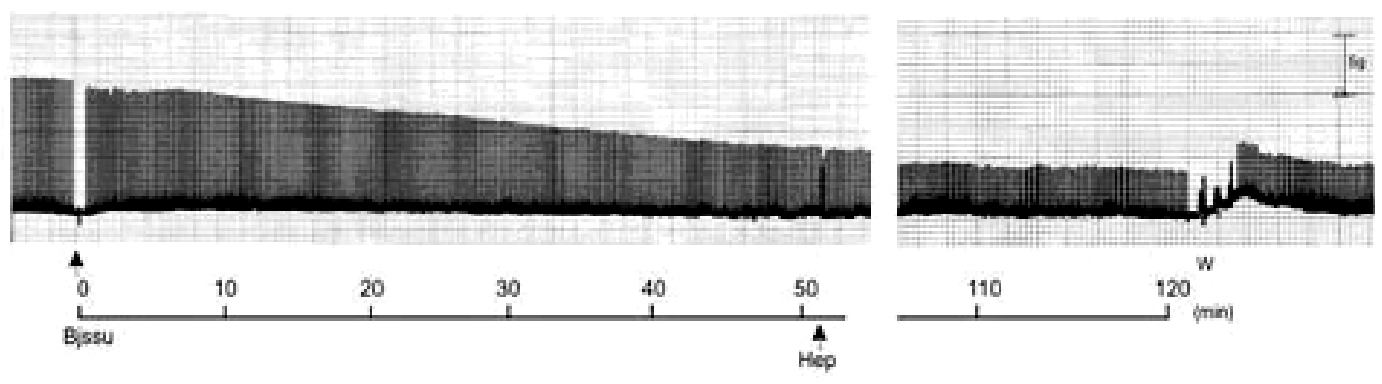

D
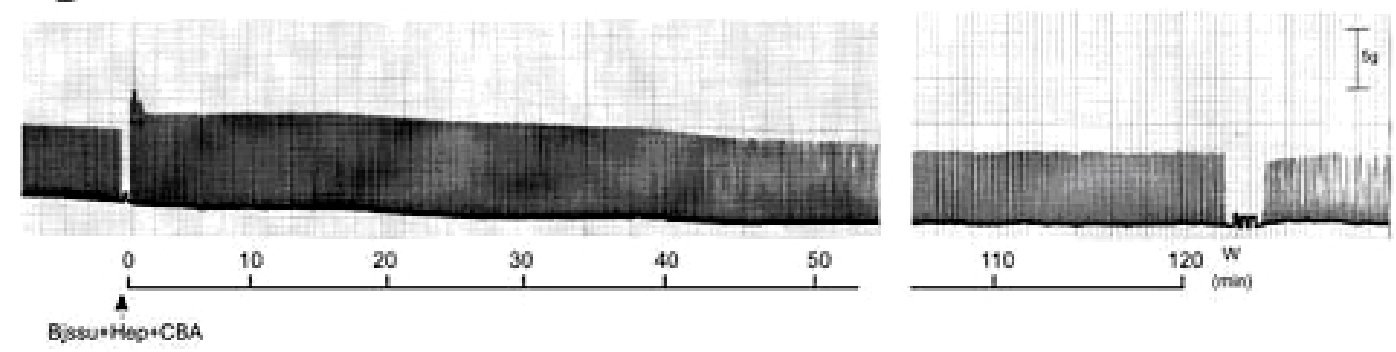

Figure 2. Record of twitch-tension in the PND after heparin and antivenom addition. (A) pretreatment with heparin $(1 \mu \mathrm{L} / \mathrm{mL})$ for 15 minutes before venom $(40 \mu \mathrm{g} / \mathrm{mL})$ addition; (B) pretreatment with CBA $(120 \mu \mathrm{L} / \mathrm{mL})$ for 15 minutes before venom addition, (C) venom treatment and heparin addition after $50 \%$ blockade; (D) Bjssu:Hep:CBA mixture $(40 \mu \mathrm{g} / \mathrm{mL}: 1 \mu \mathrm{mL} / \mathrm{mL}: 120 \mu \mathrm{L} / \mathrm{mL})$ addition incubated for 120 minutes. Note that in $\mathbf{A}, \mathbf{B}$ and $\mathbf{D}$ the heparin and antivenom showed protective effect 
against the Bjssu venom. Nevertheless, in $\mathbf{C}$, when the Bjssu effect was deflagrated and the heparin was added earlier, the heparin efficacy was reduced (W, washing).

\section{DISCUSSION}

Envenomations caused by $B$. jararacussu frequently produce local and systemic effects (16). These effects are induced by a variety of venom compounds, including myotoxic phospholipases $A_{2}$ and metalloproteinases, among other proteins and peptides (17). B. jararacussu venom is not a potent neuromuscular blocker in vivo, and is less nocive in vitro than the venoms of certain neurotoxic snakes, which contain $\alpha$ and $\beta$ neurotoxins that induce neuromuscular blockade at concentrations lower than $5 \mu \mathrm{g} / \mathrm{mL}$ (18). However, this venom also causes total neuromuscular blockade in isolated mouse preparation at doses above $50 \mu \mathrm{g} / \mathrm{mL}(5,6)$.

Heparin is a highly acidic sulfated glycosaminoglycan with a high sulfate concentration, which is able to form inactive acid-base complexes with basic myotoxins from Bothrops venoms (19-23). Due to its polyanionic nature, heparin can interact with many molecules that have cationic sites, including proteins from the extracellular matrix (fibronectin, laminae, vitronectin) $(5,19,22-24)$. Heparin can also interact with phospholipases $A_{2}$ present in many snake venoms that may or may not affect the enzymatic activity of these proteins $(25,26)$. Similar results were observed by De Oliveira et al. (27) using suramin, a polyanionic substance, that formed inactive acid-base complexes with BthTX-I.

Commercial bothropic antivenom is raised in horses and may be either monovalent (against Bothrops only) or polyvalent (against Crotalus and Bothrops or Bothrops and Lachesis muta); it is produced by mixing seven Bothrops venoms: $B$. alternatus, $B$. cotiara, B. jararaca, B. jararacussu, B. moojeni, B. neuwiedi and B. pradoi (28). The antivenom neutralizes the neuromuscular blockade caused by these venoms, evidencing the formation of an antigen-antibody complex. In this study, the antivenom $(120 \mu \mathrm{L} / \mathrm{mL})$ addition promoted a facilitative effect in isolated preparation, corroborating the findings of Oshima-Franco et al. (29), who argued that this effect is caused by the presence of a thermostable substance or may simply be a non-specific protein-concentration (excess) effect. The correct explanation for this effect remains unclear, but involves the antivenom production. Antivenom fractionation could help elucidate this phenomenon. 
Melo et al. (21) described the need for a pre-incubation with heparin and venom to form an acid-base complex. However, this procedure is unnecessary as demonstrated in the present study, since the incubation of heparin, before or after the venom addition, displayed an efficient venom-neutralizing effect. Oshima-Franco et al. (29) showed that the pre-incubation of heparin $(60 \mu \mathrm{L} / \mathrm{mL})$ with bothropstoxin-I, the principal toxin of $B$. jararacussu venom, for 30 minutes, induced $100 \%$ protection in the contractile response in mouse neuromuscular preparations.

In our experimental conditions, when examining pretreatment with heparin $(1 \mu \mathrm{L} / \mathrm{mL})$ or antivenom (120 $\mathrm{LL} / \mathrm{mL})$, for 15 minutes before venom addition, we observed a protective ability via the twitch-tension of mouse phrenic nerve-diaphragm. Under a different protocol, heparin addition after 50\% blockade (about 50 minutes after incubation) induced by the venom itself, produced a $40 \%$ contractile response after 120 minutes, demonstrating that heparin was efficient in the heparin-venom complex, even when added in the presence of neuromuscular blockade, which indicates the use of different incubation time-points.

Boechat et al. (30) found the association between heparin and antivenom to be more effective in neutralizing the lethal activity of Bothrops envenomation. In this study the mixture (heparin:antivenom) was also effective in neutralizing its neurotoxic venom effects, but no significant difference was observed when compared to heparin:venom and antivenom:venom incubations.

As examples of venom or toxin neutralization, many other substances have been assayed, ranging from manganese salt, which provides significant protection against Bjssu venom and its toxin neurotoxicity, to medicinal plants, as shown by hydroalcoholic extract of Casearia sylvestris against the neuromuscular paralyzing effect of BThTX-I (31-33). Therefore, new therapies in parallel with the antivenom treatment would be fundamental in minimizing local effects induced by snake venoms, specially those from the Bothrops genus (30).

In conclusion, this study shows that heparin and commercial bothropic antivenom were capable of neutralizing the neurotoxic effects induced by whole $B$. jararacussu venom, even when the treatment is examined at different time-points. Heparin showed a more efficient protection than antivenom, considering the lower amount required $(1 \mu \mathrm{L} / \mathrm{mL})$ in relation to antivenom $(120 \mu \mathrm{L} / \mathrm{mL})$. 


\section{ACKNOWLEDGMENTS}

We thank Gildo B. Leite for technical assistance. This study was supported by The National Council for Scientific and Technological Development (CNPq) of the Brazilian Ministry of Education.

\section{REFERENCES}

1. Secretaria do Estado da Saúde de São Paulo. Manual de Vigilância Epidemiológica. Acidentes por animais peçonhentos. Identificação, diagnóstico e tratamento. São Paulo; 2001.

2. Cardoso JLC. Bothropic accidents. Mem Inst Butantan. 1990;52(suppl):43-4.

3. Brazil V, Pestana R. Nova contribuição ao estudo do envenenamento ophidico. Rev Med São Paulo. 1909;19:21-2.

4. Queiroz LS, Santo-Neto H, Assakura MT, Reichl AP, Mandelbaum FR. Pathological changes in muscle caused by haemorrhagic and proteolytic factors from Bothrops jararaca snake venom. Toxicon. 1985:23(2):341-5.

5. Heluany NF, Homsi-Brandeburgo MI, Giglio JR, Prado-Franceschi J, RodriguesSimioni L. Effects induced by bothropstoxin, a component from Bothrops jararacussu snake venom, on mouse and chick muscle preparations. Toxicon. 1992;30(10):120310.

6. Rodrigues-Simioni L, Borgese N, Ceccarelli B. The effects of Bothrops jararacussu venom and its components on frog nerve-muscle preparation. Neuroscience. 1983;10(2):475-89.

7. Gutiérrez JM, Lomonte B. Phospholipase $A_{2}$ myotoxins from Bothrops snake venoms. Toxicon. 1995;33(11):1405-24.

8. Homsi-Brandeburgo MI, Queiroz LS, Santo-Neto H, Rodrigues-Simioni L, Giglio JR. Fractionation of Bothrops jararacussu snake venom: partial chemical characterization and biological activity of bothropstoxin. Toxicon. 1988:26(7):615-27.

9. Arni RK, Ward RJ, Cintra ACO, Giglio JR. Crystallization and preliminary diffraction data of bothropstoxin I isolated from the venom of Bothrops jararacussu. Toxicon. 1995;33(3):383-86.

10. Ministério da Saúde do Brasil. Fundação Nacional de Saúde. Manual de diagnóstico e tratamento de acidentes por animais peçonhentos. Brasília; 1998. 
11. Doin-Silva $R$, Baranauskas $V$, Rodrigues-Simioni $L$, da Cruz-Höfling MA. The ability of low level laser therapy to prevent muscle tissue damage induced by snake venom. Photochem Photobiol. 2009;85(1):63-9.

12. Kanashiro MM, de Cássia M, Escocard R, Petretski JH, Prates MV, Alves EW, et al. Biochemical and biological properties of phospholipases $A(2)$ from Bothrops atrox snake venom. Biochem Pharmacol. 2002;64(7):1179-86.

13. Soares AM, Sestito WP, Marcussi S, Stabeli RG, Andriao-Escarso SH, Cunha $A O$, et al. Alkylation of myotoxic phospholipases $A_{2}$ in Bothrops moojeni venom: a promising approach to an enhanced antivenom production. Int $\mathrm{J}$ Biochem Cell Biol. 2004;36(2):258-70.

14. Melo PA, Ownby CL. Ability of wedelolactone, heparin, and para-bromophenacyl bromide to antagonize the myotoxic effects of two crotaline venoms and their PLA 2 myotoxins. Toxicon. 1999;37(1):199-215.

15. Bülbring $\mathrm{E}$. Observations on the isolated phrenic nerve diaphragm preparation of the rat. Br J Pharmacol. 1946;1(1):38-61.

16. Milani Junior R, Jorge MT, De Campos FP, Martins FP, Bousso A, Cardoso JL, et al. Snake bites by the jararacuçu (Bothrops jararacussu): clinicopathological studies of 29 proven cases in São Paulo State, Brazil. Q J Med. 1997;90(5):323-34.

17. Gutiérrez JM, Rucavado A. Snake venom metalloproteinases: their role in the pathogenesis of local tissue damage. Biochimie. 2000;82(9-10):841-45.

18. Rodrigues L, Cunha DB, Leite GB, Borja-Oliveira CR, Cintra ACO, RodriguesSimioni L, et al. Ação da heparina contra a atividade neurotóxica da miotoxina bothropstoxina-I. Saúde Rev. 2004;6(14):19-29.

19. Melo PA, Suarez-Kurtz G. Release of creatine kinase from skeletal muscles by Bothrops jararacussu venoms: heparin potentiation of inhibition by antivenom. Braz $\mathrm{J}$ Med Biol Res. 1988;21(3):545-8.

20. Melo PA, Suarez-Kurtz G. Release of sarcoplasmic enzymes from skeletal muscles by Bothrops jararacussu venom: antagonism by heparin and by the serum of South American marsupials. Toxicon. 1988;26(1):87-95.

21. Melo PA, Homsi-Brandeburgo MI, Giglio JR, Suarez-Kurtz G. Antagonism of the myotoxic effects of Bothrops jararacussu venom and bothropstoxin by polyanions. Toxicon. 1993;31(3):285-91. 
22. Lomonte B, Moreno E, Tarkowski A, Hanson LA, Maccarana M. Neutralizing interaction between heparins and myotoxin II, a lysin 49 phospholipase $A_{2}$ from Bothrops asper snake venom. J Biol Chem. 1994;269:29867-73.

23. Lomonte B, Tarkowski A, Bagge V, Hanson LA. Neutralization of the cytolytic and myotoxic activities of phospholipases $A_{2}$ from Bothrops asper snake venom by glycosaminoglycans of the heparin/heparan sulfate family. Biochem Pharmacol. 1994;47(9):1509-18.

24. Lane DA, Adams L. Non-anticoagulant uses of heparin. $\mathrm{N}$ Engl J Med. 1993;329(2):129-30.

25. Diccianini M, Mistry MJ, Hug K, Harmony JA. Inhibition of phospholipase $A_{2}$ by heparin. Biochem Biophys Acta. 1990;1046(3):242-48.

26. Condreas E, De Vries A, Mager J. Hemolysis and splitting of human erythrocyte phospholipids by snake venoms. Biochim Biophys Acta. 1964;84:60-73.

27. De Oliveira M, Cavalcante WLG, Arruda EZ, Melo PA, Dal-Pai Silva M, Gallacci M. Antogonism of myotoxic and paralyzing activities of bothropstoxin-I by suramin. Toxicon 2003; 42(4):373-9.

28. Dos-Santos MC, Gonçalves LR, Fortes-Dias CL, Cury Y, Gutiérrez JM, Furtado MF. The efficacy of the bothropic-crotalic antivenom in the neutralization of the main Bothrops jararacussu venom activities. Rev Inst Med Trop São Paulo. 1992;34(2):7783.

29. Oshima-Franco Y, Leite GB, Silva GH, Cardoso DF, Hyslop S, Giglio JR, et al. Neutralization of the pharmacological effects of bothropstoxin-I from Bothrops jararacussu (jararacuçu) venom by crotoxin antiserum and heparin. Toxicon. 2001;39(10):1477-85.

30. Boechat AL, Paiva CS, França FO, Dos-Santos MC. Heparin-antivenom association: differential neutralization effectiveness in Bothrops atrox and Bothrops erythromelas envenoming. Rev Inst Med Trop São Paulo. 2001;43(1):7-14.

31. Bueno LGF, Leite GB, Cruz-Hofling MA, Rodrigues-Simioni L, Oshima-Franco Y. Effects of manganese $\left(\mathrm{Mn}^{2+}\right)$ on neurotoxic and myotoxic activities induced by Crotalus durissus terrificus and Bothrops jararacussu venoms in chick biventer cervicis preparations. J Venom Anim Toxins incl Trop Dis. 2007;13(2):479-99. 
32. Oshima-Franco Y, Leite GB, Belo CA, Hyslop S, Prado-Franceschi J, Cintra AC, Giglio JR, da Cruz-Hofling MA, Rodrigues-Simioni L. The presynaptic activity of bothropstoxin-I, a myotoxin from Bothrops jararacussu snake venom. Basic Clin Pharmacol Toxicol 2004;95(4):175-82.

33. Oshima-Franco Y, Alves CMV, Andréo Filho N, Gerenutti M, Cintra ACO, Leite GB, Rodrigues-Simioni L, Silva MG. Neutralization of the neuromuscular activity of Bothropstoxin-I, a myotoxin from Bothrops jararacussu snake venom, by a hidroalcoholic extract of Casearia sylvestris Sw (guaçatonga). J Venom Anim Toxins incl Trop Dis. 2005;11(4):465-68. 